\title{
Adesão às práticas seguras na atenção ao parto
}

\author{
Adherence to safety childbirth care practices
}

\author{
Luana Sousa de Carvalho' • Isabelly Costa Lima de Oliveira ${ }^{2} \bullet$ Raphaele Maria Almeida Silva ${ }^{3}$ \\ Taynan da Costa Alves ${ }^{4} \bullet$ Fernanda Maria Carvalho Fontenele $^{5} \bullet$ Rhanna Emanuela Fontenele Lima Carvalho $^{6}$
}

\begin{abstract}
RESUMO
Objetivo: Investigar a adesão às práticas seguras, pelos profissionais da saúde, durante o parto. Método: Estudo descritivo, transversal de abordagem quantitativa, desenvolvido nos meses de agosto a outubro de 2018, em um hospital de nível terciário e de ensino no Nordeste brasileiro. Participaram da pesquisa os profissionais da saúde, de plantão na sala de parto, durante o período da coleta dos dados.A coleta de dados foi realizada por meio do Instrumento de Observação para Partos Seguros da OMS para monitorizar a aplicação das práticas essenciais antes da expulsão e logo após o parto. Resultados: Os resultados permitiram identificar taxas satisfatórias de boas práticas de segurança do paciente conforme recomendado pela Organização Mundial da Saúde. Considerou-se que há atitudes profissionais favoráveis à verificação de Segurança no Parto o que resulta de forma direta na redução de eventos adversos evitáveis na assistência materna e perinatal. Conclusão: $O$ instrumento destacou-se como uma estratégia que pode fortalecer a cultura de segurança do paciente na instituição, pois permite trabalhar mais a adesão dos profissionais às práticas seguras, e requer contínuo treinamento dos profissionais para melhores resultados.

Palavras chave: Serviços de Saúde Materno-Infantil; Segurança do Paciente; Estudo Observacional;Time Out na Assistência à Saúde; Lista de Checagem.
\end{abstract}

\begin{abstract}
Objective: To investigate adherence to safe practices by health professionals during childbirth. Method: This is a descriptive cross-sectional study with a quantitative approach, conducted from August to October 2018, in a tertiary and teaching hospital in Northeastern Brazil. The study included health professionals on duty in the delivery room during the period of data collection. Data collection was performed through the WHO Safe Birth Observation Instrument to monitor the application of essential practices before expulsion and soon after delivery. Results: The results allowed us to identify satisfactory rates of good patient safety practices as recommended by the World Health Organization. It was considered that there are professional attitudes favorable to the Safety Check in Childbirth which directly results in the reduction of preventable adverse events in the child. maternal and perinatal care. Conclusion:The instrument stood out as a strategy that can strengthen the patient safety culture in the institution, as it allows working more adherence of professionals to safe practices, and requires continuous training of professionals for better results.
\end{abstract}

Keywords: Maternal and Child Health Services; Patient safety; Observational study; Time Out in Health Care; Check list.

I Residente em Enfermagem Obstétrica da Universidade Estadual do Ceará. Membro do Grupo de Pesquisa Segurança,Tecnologia e Cuidados Clínicos em Enfermagem e Saúde (SETECC - UECE).

2 Mestrado pelo Programa de Pós-Graduação em Cuidados Clínicos da Universidade Estadual do Ceará. Especialista em Enfermagem Pediátrica e Neonatal pela Faculdade Metropolitana da Grande Fortaleza. Membro do Grupo de Pesquisa Segurança, Tecnologia e Cuidados Clínicos (SETECC - UECE).

3 Enfermeira pela Universidade Estadual do Ceará. Membro do Grupo de Pesquisa em Segurança do Paciente, Tecnologia e Cuidados Clínicos (SETECC - UECE).

4 Enfermeira pela Universidade Estadual do Ceará. Membro do Grupo de Pesquisa de Segurança do Paciente, Tecnologia e Cuidados Clínicos (SETECC - UECE).

5 Mestrado pelo Programa de Pós-Graduação em Cuidados Clínicos em Enfermagem e Saúde da Universidade Estadual do Ceará. Especialista em Enfermagem Obstétrica, em Gestão da Qualidade em Ambientes Hospitalares e em Enfermagem Pediátrica e Neonatal.

6 Doutorado em Enfermagem Fundamental da Universidade de São Paulo. Membro da Rede Brasileira de Segurança do Paciente (Ceará) e da Sociedade Brasileira de Segurança do Paciente (SOBRASP). Líder do Grupo de Pesquisa Segurança, Tecnologia e Cuidados Clínicos (SETECC - UECE). 


\section{INTRODUÇÃO}

A segurança do paciente tem se tornado uma temática discutida em todo mundo, porém muitas práticas hospitalares ainda devem ser revistas e/ou modificadas, buscando cada vez mais proporcionar uma assistência segura, tornando-se parte fundamental dos processos relacionados à melhoria da qualidade assistencial. Com base nisso, é importante enfatizar a garantia do direito à maternidade segura, que continua sendo um problema relevante e atinge milhares de mulheres anualmente ${ }^{(1)}$.

Nesse contexto, ressalta-se que o parto é acompanhado de diversos significados e representações, pois é considerado um acontecimento esperado e idealizado em que mãe e filho devem ser os protagonistas, o que o torna um episódio complexo, especial e marcante na vida da mulher (2). Diante disso, a assistência adequada no momento do parto representa um passo indispensável para garantir que ela possa vivenciar a experiência da maternidade com segurança e bem-estar, sendo este um direito fundamental de toda mulher ${ }^{(3)}$.

Com isso, considera-se que o índice elevado de casos relacionados à morte materna é um problema a ser enfrentado em todo o mundo. Segundo a Organização Mundial de Saúde (OMS), o Brasil está inserido no grupo de países latino-americanos que conseguiram atingir grandes avanços em relação a redução de mortes relacionadas à gravidez ou parto no período de 1990 a 2013. Neste mesmo período, o país reduziu sua taxa de mortalidade materna em $43 \%$. Entretanto, a OMS alerta que apesar do Brasil ter alcançado esse número significativo, este progresso não foi suficiente para atingir o objetivo de redução de $75 \%$, estabelecido pelos Objetivos de Desenvolvimento do Milênio (ODM) ${ }^{(4)}$.

Nesse âmbito, devido ao elevado número de morbimortalidade materno infantil, bem como, a discussão mundial nas últimas décadas em relação à segurança do paciente, foram criadas intervenções em busca de contornar esse cenário. Uma delas foi à elaboração da Lista de Verificação da OMS para Partos Seguros, com recomendações baseadas em evidência científica, criada com o objetivo de apoiar o uso de práticas essenciais de cuidados maternos e perinatais ${ }^{(5)}$.

Nesse sentido, o presente estudo justifica-se devido às altas taxas de mortalidade materna infantil, e também devido, ainda hoje, serem utilizadas práticas intervencionistas desnecessárias durante o parto, para acelerar o seu desenvolvimento, comprometendo assim, a segurança e o sucesso desse parto e a vida da mãe e do bebê. Diante disso, o objetivo do presente estudo consistiu em investigar a adesão às práticas seguras, pelos profissionais da saúde, durante o parto.

\section{MÉTODO}

Trata-se de uma pesquisa descritiva, transversal de abordagem quantitativa, desenvolvida na sala de parto e no centro cirúrgico de um hospital de nível terciário de alta complexidade e de ensino no Nordeste brasileiro.

A amostra da pesquisa foi definida por conveniência e composta por 40 partos. Os partos observados foram realizados por profissionais de diferentes categorias, médicos, enfermeiros, residentes e internos de medicina e de enfermagem, supervisionados pelo profissional de sua categoria na sala de parto. Desta forma, compuseram a amostra todos os partos realizados durante o período de coleta, no período da manhã e tarde nos meses de agosto a outubro de 2018.

A equipe de coleta da pesquisa foi composta pela pesquisadora e mais duas acadêmicas de enfermagem, que passaram por um treinamento de 12 horas antes do início da coleta. Inicialmente o profissional responsável pela condução do parto era abordado pelas pesquisadoras e convidado a participar da pesquisa. Ressalta-se que, devido à pesquisa tratar de um estudo observacional, em nenhum momento as pesquisadoras interferiram nas práticas dos profissionais. Para evitar a mudança ativa de comportamento dos profissionais quando submetidos a estudos observacionais, as primeiras análises realizadas no período de uma semana foram descartadas.

O instrumento utilizado para a coleta de dados foi o Instrumento de Observação da Lista de Verificação para Partos Seguros da OMS para monitorizar a aplicação das práticas essenciais antes da expulsão e logo após o parto ${ }^{(6)}$. A Lista de Verificação foi desenvolvida com base em evidências científicas e organizada em quatro diferentes pontos de pausa ou momentos de observação. $O$ ponto de pausa I é realizado na admissão:A parturiente é examinada no momento da admissão, confirmar se a mãe necessita ser transferida para outra unidade, para prepará-la, e ao seu acompanhante, para o trabalho de parto e o parto. O ponto de pausa 2 é realizado antes da expulsão (ou antes da cesariana). A mulher é examinada nesse momento para detectar e tratar complicações que possam acontecer durante o trabalho de parto e prepará-la para eventos de rotina e possíveis situações de crise que possam ocorrer depois do parto. $O$ ponto de pausa 3 é realizado logo após o nascimento (no espaço de uma hora). Nesse momento é importante realizar a pausa para examinar a mãe e o bebê, com o objetivo de detectar e tratar complicações que possam ocorrer depois do parto e orientar a mãe e o seu acompanhante sobre os sinais de perigo para os quais devem pedir ajuda. Por fim, o ponto de pausa 4 que é realizado antes da alta. Nesse ponto de pausa, verifica-se se mãe e o bebê estão saudáveis e se está tudo preparado para a alta.

Os momentos observados pela pesquisadora foram os momentos 2 (19 itens): Antes da expulsão (ou antes 
da cesariana) e o momento de pausa 3 (I5 itens): Logo após o nascimento (no espaço de uma hora). $O$ preenchimento das questões segue uma resposta dicotômica do tipo $\operatorname{Sim}(\mathrm{S})$ ou Não $(\mathrm{N})^{(6)}$.

A taxa de adesão foi calculada pelo total de repostas esperadas sobre o total de partos observados. Foi considerada uma taxa de adesão satisfatória, quando determinada prática esperada fosse realizada por todos os profissionais (100\%) observados.

O projeto foi aprovado pelo Comitê de Ética em Pesquisa da Universidade Estadual do Ceará e possui número CAAE 893।38I8.3.0000.5534 e parecer número 2.687.016.

\section{RESULTADOS}

Durante os três meses do estudo, foram observados 40 partos realizados por profissionais de diferentes categorias. Destes, 25 (62,5\%) foram partos vaginais. Os residentes realizaram 22(55\%) dos partos, destes $12(30 \%)$ eram residentes de enfermagem obstétrica.

Quanto a observação da adesão dos profissionais às práticas seguras, os resultados foram divididos em duas tabelas, cada uma categorizada por tipo de parto (cesáreo e vaginal) e por período do parto (antes e após o parto). A tabela I contém informações em relação à taxa de adesão das práticas avaliadas antes do parto cesáreo e do parto vaginal.

TABELA 1 - Taxas de adesão das práticas avaliadas antes do parto cesáreo $(N=15)$ e do parto vaginal ( $n=25)$, Fortaleza, Ceará, Brasil, 2018

\begin{tabular}{|c|c|c|c|c|c|c|c|c|}
\hline \multirow{3}{*}{ Itens } & \multicolumn{4}{|c|}{ Antes do parto cesáreo(n=15) } & \multicolumn{4}{|c|}{ Antes do parto vaginal $(n=25)$} \\
\hline & \multicolumn{2}{|c|}{ Respostas Observadas } & \multirow{2}{*}{$\begin{array}{l}\text { Respostas } \\
\text { Esperadas }\end{array}$} & \multirow{2}{*}{$\begin{array}{c}\text { Taxa de } \\
\text { Adesão } \\
(\%)\end{array}$} & \multicolumn{2}{|c|}{$\begin{array}{l}\text { Respostas } \\
\text { Observadas }\end{array}$} & \multirow{2}{*}{$\begin{array}{l}\text { Respostas } \\
\text { Esperadas }\end{array}$} & \multirow{2}{*}{$\begin{array}{c}\text { Taxa de } \\
\text { Adesão } \\
(\%)\end{array}$} \\
\hline & $\begin{array}{l}\text { Sim } \\
n(\%)\end{array}$ & $\begin{array}{l}\text { Não } \\
n(\%)\end{array}$ & & & $\begin{array}{l}\text { Sim } \\
\mathrm{n}(\%)\end{array}$ & $\begin{array}{l}\text { Não } \\
\mathrm{n}(\%)\end{array}$ & & \\
\hline $\begin{array}{l}\text { 1.A mãe precisa tomar } \\
\text { antibióticos? }\end{array}$ & $15(100)$ & 0 & Sim & 100 & $5(20)$ & $\begin{array}{c}20 \\
(80)\end{array}$ & Não & 80 \\
\hline $\begin{array}{l}\text { 1a- } A \text { temperatura foi } \\
\text { medida? }\end{array}$ & $15(100)$ & 0 & Sim & 100 & $\begin{array}{c}14 \\
(56)\end{array}$ & $\begin{array}{c}11 \\
(44)\end{array}$ & Sim & 56 \\
\hline $\begin{array}{l}\text { 2.A mãe precisa de } \\
\text { sulfato de magnésio e de } \\
\text { tratamento hipertensivo? }\end{array}$ & $7(46,7)$ & $8(53,3)$ & Não & 53,3 & 0 & $\begin{array}{c}25 \\
(100)\end{array}$ & Não & 100 \\
\hline $\begin{array}{l}\text { 2a.A pressão arterial foi } \\
\text { medida? }\end{array}$ & $15(100)$ & 0 & Sim & 100 & $\begin{array}{c}17 \\
(68)\end{array}$ & $\begin{array}{c}8 \\
(32)\end{array}$ & Sim & 68 \\
\hline \multicolumn{9}{|l|}{$\begin{array}{l}\text { 3.Os materiais essenciais } \\
\text { estão ao lado da cama? }\end{array}$} \\
\hline \multicolumn{9}{|l|}{ Para a mãe: } \\
\hline 3a- Luvas & $15(100)$ & 0 & Sim & 100 & $\begin{array}{c}24 \\
(96)\end{array}$ & $\begin{array}{c}1 \\
(4) \\
\end{array}$ & Sim & 96 \\
\hline $\begin{array}{l}\text { 3b- Antisséptico de mãos à } \\
\text { base de álcool ou sabão e } \\
\text { água limpa }\end{array}$ & $14(93,3)$ & $1(6,7)$ & Sim & 93,3 & $\begin{array}{c}14 \\
(56)\end{array}$ & $\begin{array}{c}11 \\
(44)\end{array}$ & Sim & 56 \\
\hline $\begin{array}{l}\text { 3c- Ocitocina, } 10 \text { unidades } \\
\text { na seringa }\end{array}$ & $15(100)$ & 0 & Sim & 100 & $\begin{array}{c}24 \\
(96)\end{array}$ & $1(4)$ & Sim & 96 \\
\hline \multicolumn{9}{|l|}{ Para o bebê: } \\
\hline 3a- Toalha limpa & $15(100)$ & 0 & Sim & 100 & $\begin{array}{c}25 \\
(100)\end{array}$ & 0 & Sim & 100 \\
\hline $\begin{array}{l}\text { 3b - Lâmina/tesoura } \\
\text { esterilizada para cortar o } \\
\text { cordão }\end{array}$ & $15(100)$ & 0 & Sim & 100 & $\begin{array}{c}25 \\
(100)\end{array}$ & 0 & Sim & 100 \\
\hline 3c- Dispositivo de aspiração & $15(100)$ & 0 & Sim & 100 & $24(96)$ & $1(4)$ & Sim & 96 \\
\hline 3d- Balão e máscara & $15(100)$ & 0 & Sim & 100 & $24(96)$ & $1(4)$ & Sim & 96 \\
\hline $\begin{array}{l}\text { 4- Uso correto do material } \\
\text { essencial: }\end{array}$ & & & & & & & & \\
\hline $\begin{array}{l}\text { 4a- As mãos foram lavadas } \\
\text { imediatamente após o } \\
\text { parto? }\end{array}$ & $9(60)$ & $6(40)$ & Sim & 60 & $19(76)$ & $6(24)$ & Sim & 76 \\
\hline
\end{tabular}




\begin{tabular}{|c|c|c|c|c|c|c|c|c|}
\hline $\begin{array}{l}\text { 4b- Foram usadas luvas } \\
\text { durante o parto? }\end{array}$ & $15(100)$ & 0 & Sim & 100 & $24(96)$ & $1(4)$ & Sim & 96 \\
\hline $\begin{array}{l}\text { 4c- Foram dadas } 10 \\
\text { unidades de Oxitocina à } \\
\text { mãe um minuto após o } \\
\text { parto? }\end{array}$ & $15(100)$ & 0 & Sim & 100 & $20(80)$ & $5(20)$ & Sim & 80 \\
\hline $\begin{array}{l}\text { 4d- A respiração do bebê foi } \\
\text { avaliada? }\end{array}$ & $\begin{array}{c}15 \\
(100)\end{array}$ & 0 & Sim & 100 & $\begin{array}{c}24 \\
(96)\end{array}$ & $\begin{array}{c}1 \\
(4)\end{array}$ & Sim & 96 \\
\hline $\begin{array}{l}\text { *4e- Se o bebê não } \\
\text { respirava, tentou-se } \\
\text { desobstruir as vias } \\
\text { respiratórias? }\end{array}$ & - & - & Sim & - & - & - & Sim & - \\
\hline $\begin{array}{l}\text { 5- Foi identificado e } \\
\text { disponibilizado um } \\
\text { assistente para ajudar } \\
\text { no parto, em caso de } \\
\text { necessidade? }\end{array}$ & $\begin{array}{c}15 \\
(100)\end{array}$ & 0 & Sim & 100 & $\begin{array}{c}25 \\
(100)\end{array}$ & 0 & Sim & 100 \\
\hline
\end{tabular}

Diante do exposto, os resultados apontam que as práticas antes do parto cesáreo obtiveram uma taxa de adesão variando entre $60 \%$ a $100 \%$. Dos 19 itens, 13 apresentaram taxa de $100 \%$ de adesão às práticas. Ressalta-se que a prática $4 a$, que avalia a higienização das mãos após o parto, observou-se $60 \%$ de adesão (Tabela I).

Vale salientar que os itens sobre a administração sulfato de magnésio e tratamento hipertensivo, a maioria das mulheres não precisou desse tratamento, mas todas que necessitaram, foram atendidas. Assim como o item de administração de antibióticos no parto vaginal. A recomendação da OMS é que esse tipo de intervenção ocorra antes do parto cesariano e no parto vaginal em alguns casos é recomendável.

A taxa de adesão das práticas antes do parto vaginal, variou entre $56 \%$ a $100 \%$. Destacam-se quatro práticas com 100\% de adesão. Em contrapartida, as ações com baixa adesão após o parto vaginal, referem-se a verificação da temperatura e quanto ao material essencial, tipo álcool gel ou sabão, próximo ao leito. Além disso, observa-se que o item 4a, relacionado a higienização das mãos após o parto, não atingiu a porcentagem considerada como parâmetro para uma boa taxa de adesão profissional, sendo esperado 100\% de adesão para essa ação. (Tabela I).

Em relação às práticas após o parto cesáreo, a taxa de adesão variou entre $6,6 \%$ a $100 \%$, exceto para os itens sobre amamentação no espaço de I hora, que não obteve adesão de nenhum profissional. Além disso, somente um profissional explicou os sinais de perigo para a mãe e o acompanhante (Tabela 2).

No que se refere a adesão às práticas após o parto vaginal, constatou-se que a taxa variou entre $28 \%$ a $100 \%$ para todas as práticas. No entanto somente duas práticas obtiveram $100 \%$ de adesão. A prática com menos adesão dos profissionais foi em relação a verificação da temperatura e os "Os sinais de perigo foram explicados à mãe/acompanhante?", com 10 (40\%) e 7 (28\%) de adesão, respectivamente (Tabela 2 ).

\section{DISCUSSÃO}

Dos 17 itens e subitens avaliados antes do parto, 13 atingiram a taxa de adesão de $100 \%$ no parto cesáreo e quatro no parto vaginal. Dentre os 15 itens e subitens avaliados após o parto, sete atingiram uma taxa de adesão de $100 \%$ no parto cesáreo e duas no parto vaginal. Diante disso, das 32 práticas avaliadas antes e após o parto, 22 obtiveram uma taxa de adesão de $100 \%$.

A Lista de Verificação de Segurança no Parto corresponde a um documento recente, por esse motivo, muitos estudos que caracterizam sua implantação, adesão e repercussão na qualidade da assistência ainda estão em andamento em diversos países. Um dos estudos pioneiros desenvolvido em Karnataka, na Índia, demonstrou uma taxa de adesão de $95 \%$ dos profissionais de saúde ao uso da lista de verificação, além de registrar um aumento de práticas essenciais de 10 para 25 , dos 29 itens. ${ }^{(7)}$

Quanto ao tipo de parto, no presente estudo foi observado um maior número de partos vaginais 25 (62,5\%). No Sistema Único de Saúde (SUS), de modo geral, a indicação de uma cesariana é realizada mediante o diagnóstico de intercorrências durante a gestação ou trabalho de parto havendo possibilidade limitada de agendamento de cesariana a pedido da mulher ${ }^{(8)}$. Embora o parto vaginal tenha apresentado maior frequência, o hospital de estudo obteve uma taxa ainda elevada de cesarianas (37,5\%) diferente do que é preconizado pela OMS, no qual orienta que as taxas de cesariana não ultrapassem os $15 \%$ dos $\operatorname{partos}^{(9)}$. 
TABELA 2 - Taxa de adesão das práticas avaliadas após o parto cesáreo (N=15), Fortaleza, Ceará, Brasil, 2018

Após parto cesáreo $(n=15)$

Após parto vaginal $(n=25)$

Itens

\begin{tabular}{|c|c|c|c|}
\hline \multicolumn{2}{|c|}{$\begin{array}{l}\text { Respostas } \\
\text { Observadas }\end{array}$} & \multirow{2}{*}{$\begin{array}{l}\text { Respostas } \\
\text { Esperadas }\end{array}$} & \multirow{2}{*}{$\begin{array}{c}\text { Taxa de } \\
\text { Adesão } \\
(\%)\end{array}$} \\
\hline Sim & Não & & \\
\hline $\begin{array}{l}N \\
(\%)\end{array}$ & $\begin{array}{l}N \\
(\%)\end{array}$ & & \\
\hline
\end{tabular}

\begin{tabular}{|c|c|c|c|c|c|c|c|c|}
\hline & (\%) & (\%) & & & $(\%)$ & (\%) & & \\
\hline $\begin{array}{l}\text { 1- A mãe tem sangramento } \\
\text { anormal? }\end{array}$ & 0 & $\begin{array}{c}15 \\
(100)\end{array}$ & Não & 100 & $\begin{array}{c}1 \\
(4)\end{array}$ & $\begin{array}{c}24 \\
(96)\end{array}$ & Não & 96 \\
\hline $\begin{array}{l}\text { 1a- A parteira verificou se havia } \\
\text { hemorragia? }\end{array}$ & $\begin{array}{c}15 \\
(100)\end{array}$ & 0 & Sim & 100 & $\begin{array}{c}23 \\
(92)\end{array}$ & $\begin{array}{c}2 \\
(8)\end{array}$ & Sim & 92 \\
\hline $\begin{array}{l}\text { 2- A mãe precisa tomar } \\
\text { antibióticos? }\end{array}$ & $\begin{array}{c}1 \\
(6,7)\end{array}$ & $\begin{array}{c}14 \\
(93,3)\end{array}$ & Não & 93,3 & $\begin{array}{c}1 \\
(4)\end{array}$ & $\begin{array}{c}24 \\
(96)\end{array}$ & Não & 96 \\
\hline 2a- A temperatura foi medida? & $\begin{array}{c}15 \\
(100)\end{array}$ & 0 & Sim & 100 & $\begin{array}{c}10 \\
(40)\end{array}$ & $\begin{array}{c}15 \\
(60)\end{array}$ & Sim & 40 \\
\hline $\begin{array}{l}\text { 3- A mãe precisa de sulfato } \\
\text { de magnésio e de tratamento } \\
\text { hipertensivo? }\end{array}$ & $\begin{array}{c}6 \\
(40)\end{array}$ & $\begin{array}{c}9 \\
(60)\end{array}$ & Não & 60 & 0 & $\begin{array}{c}25 \\
(100)\end{array}$ & Não & 100 \\
\hline $\begin{array}{l}\text { 3a- A pressão arterial foi } \\
\text { medida? }\end{array}$ & $\begin{array}{c}15 \\
(100)\end{array}$ & 0 & Sim & 100 & $\begin{array}{c}13 \\
(52)\end{array}$ & $\begin{array}{c}12 \\
(48)\end{array}$ & Sim & 52 \\
\hline $\begin{array}{l}\text { 4- O bebê precisa ser } \\
\text { transferido? }\end{array}$ & $\begin{array}{c}2 \\
(13,4)\end{array}$ & $\begin{array}{c}13 \\
(86,6)\end{array}$ & Não & 86,6 & $\begin{array}{c}6 \\
(24)\end{array}$ & $\begin{array}{c}19 \\
(76)\end{array}$ & Não & 76 \\
\hline $\begin{array}{l}\text { 5- } O \text { bebê precisa de } \\
\text { antibióticos? }\end{array}$ & 0 & $\begin{array}{c}15 \\
(100)\end{array}$ & Não & 100 & 0 & $\begin{array}{c}25 \\
(100)\end{array}$ & Não & 100 \\
\hline $\begin{array}{l}\text { 5a- A temperatura do bebê foi } \\
\text { medida? }\end{array}$ & $\begin{array}{c}15 \\
(100)\end{array}$ & 0 & Sim & 100 & $\begin{array}{c}23 \\
(92)\end{array}$ & $\begin{array}{c}2 \\
(8)\end{array}$ & Sim & 92 \\
\hline $\begin{array}{l}5 \mathrm{~b}-\mathrm{A} \text { frequência respiratória do } \\
\text { bebê foi medida? }\end{array}$ & $\begin{array}{c}15 \\
(100)\end{array}$ & 0 & Sim & 100 & $\begin{array}{c}24 \\
(96)\end{array}$ & $\begin{array}{c}1 \\
(4)\end{array}$ & Sim & 96 \\
\hline $\begin{array}{l}\text { 6- O bebê precisa de cuidados } \\
\text { especiais e de monitorização? }\end{array}$ & $\begin{array}{c}3 \\
(20)\end{array}$ & $\begin{array}{c}12 \\
(80)\end{array}$ & Não & 80 & $\begin{array}{c}8 \\
(32)\end{array}$ & $\begin{array}{c}17 \\
(68)\end{array}$ & Não & 68 \\
\hline 6a- $O$ bebê foi pesado? & $\begin{array}{c}14 \\
(93,3)\end{array}$ & $1(6,7)$ & Sim & 93,3 & $\begin{array}{c}24 \\
(96)\end{array}$ & $\begin{array}{c}1 \\
(4)\end{array}$ & Sim & 96 \\
\hline $\begin{array}{l}\text { 7- A amamentação começou no } \\
\text { espaço de } 1 \text { hora? }\end{array}$ & 0 & $15(100)$ & Sim & 0 & $\begin{array}{c}16 \\
(64)\end{array}$ & $\begin{array}{c}9 \\
(36)\end{array}$ & Sim & 64 \\
\hline $\begin{array}{l}\text { 8- O bebê foi colocado em } \\
\text { contato pele com pele no peito } \\
\text { ou abdômen da mãe? }\end{array}$ & $\begin{array}{c}4 \\
(26,6)\end{array}$ & $\begin{array}{c}11 \\
(73,4)\end{array}$ & Sim & 26,6 & $\begin{array}{c}23 \\
(92)\end{array}$ & $\begin{array}{c}2 \\
(8)\end{array}$ & Sim & 92 \\
\hline $\begin{array}{l}\text { 9- Os sinais de perigo } \\
\text { foram explicados à mãe/ } \\
\text { companheiro? }\end{array}$ & $\begin{array}{c}1 \\
(6,6)\end{array}$ & $\begin{array}{c}14 \\
(93,4)\end{array}$ & Sim & 6,6 & $\begin{array}{c}7 \\
(28)\end{array}$ & $\begin{array}{c}18 \\
(72)\end{array}$ & Sim & 28 \\
\hline
\end{tabular}

No presente estudo, os profissionais que realizaram os partos, independente do tipo, demonstraram uma boa adesão à maioria das práticas avaliadas no instrumento da OMS. Dentre as práticas que obtiveram 100\% de adesão antes do parto cesáreo, destaca-se a que avalia a necessidade de iniciar antibioticoterapia para a mãe. Os antibióticos previnem e tratam as infecções e deverão ser administrados em todos os partos cesarianos, além de ser administrados no parto normal quando a temperatura da mãe for igual ou superior a $38^{\circ} \mathrm{C}$, presença de corrimento vaginal fétido ou rotura do saco amniótico há mais de 18 horas. ${ }^{(7)}$

O estudo transversal realizado em maternidades com
855 parturientes evidenciou que $89,1 \%$ das que foram tratadas com regime profilático (antibioticoterapia), apenas 6, $1 \%$ desenvolveram infecção puerperal, taxa menor do que a encontrada entre mulheres que não foram tratadas $(13,0 \%)(p=0,012)$, demonstrando a efetividade do uso de antibioticoprofilaxia no parto cesáreo ao reduz o risco de infecção puerperal grave ${ }^{(10)}$.

Os itens que estão relacionados a verificação dos sinais vitais (pressão e temperatura), também atingiram $100 \%$ de adesão na cesariana, pois todas as mulheres são monitorizadas no centro cirúrgico. O pré-operatório é um momento que pode acarretar ansiedade, dúvidas e insegurança, por isso, as intervenções de enfermagem 
devem atender a todas as necessidades psicológicas (esclarecimento de dúvidas) independente do tipo de parto, verificar sinais vitais, pesar a paciente, colher material para exames conforme solicitação médica, observar higiene oral e corporal antes de encaminhar a paciente, esvaziar a bexiga 30 minutos antes da cirurgia, retirar próteses dentárias, joias, ornamentos e identificá-los e encaminhar o paciente ao centro cirúrgico (11).

Quanto aos materiais essenciais, no parto cesáreo notou-se uma taxa de adesão de $100 \%$ para os seguintes materiais, luvas, ocitocina ( 10 unidades na seringa), toalha limpa, lâmina/tesoura esterilizada para cortar o cordão e dispositivo de aspiração. Em contrapartida, no parto normal, as taxas de $100 \%$ de adesão foram atingidas apenas para toalha limpa e lâmina/tesoura esterilizada.

Em comparação a esses achados, um estudo observacional prospectivo realizado no Sri Lanka, com 824 profissionais sobre a implementação do programa de lista de verificação de parto seguro da OMS, evidenciou que $72 \%$ dos profissionais aderiram a verificação da frequência do item que está relacionado a presença e preparo dos materiais essenciais para o parto ${ }^{(12)}$.

Com base nisso, no início da expulsão ou antes da cesariana, os profissionais de saúde deverão confirmar a disponibilidade dos seguintes produtos essenciais ao lado da mãe e prontos a usar no momento do nascimento: luvas, sabão e água limpa com toalhas descartáveis ou antissépticos para as mãos à base de álcool e ocitocina ( 10 unidades internacionais numa seringa). Sendo o uso de luvas, sabão, água limpa e toalhas descartáveis, bem como, antissépticos para as mãos à base de álcool destinam-se a garantir uma boa prática de higiene das mãos durante o parto para evitar a infecção da mãe e do bebé, bem como o uso de ocitocina para ajudar na contração do útero ${ }^{(6)}$.

O uso de luvas estéreis durante o parto foi uma outra prática que obteve $100 \%$ de adesão no parto cesáreo. Além da antissepsia cirúrgica das mãos, a utilização de luvas cirúrgicas estéreis é, também, uma medida essencial para a prevenção das infecções, pois propicia uma barreira física para os micro-organismos presentes nas mãos dos profissionais de saúde, no ambiente e nos pacientes. Sendo assim, as luvas estéreis são usadas de forma complementar à antissepsia cirúrgica das mãos, sendo ambas igualmente imprescindíveis ${ }^{(12)}$.

Quanto ao uso da ocitocina um minuto após o parto cesáreo, os resultados também apontaram uma taxa de adesão de $100 \%$. A ocitocina (I0 UI, IM / IV) é um medicamento uterotônico recomendado para a prevenção da hemorragia pós-parto (HPP) no terceiro período do parto ${ }^{(7)}$.A hemorragia pós-parto (HPP) é uma emergência obstétrica considerada a causa mais comum de morte materna, sendo caracterizada pela perda de mais de $\mathbf{5 0 0}$ $\mathrm{ml}$ de sangue nas primeiras 24 horas após o parto vaginal e mais de $1000 \mathrm{ml} \mathrm{I}$ após operação cesariana ${ }^{(13)}$.

A prática de verificação da respiração do bebê após o parto cesáreo também obteve $100 \%$ de taxa de adesão pelos profissionais. No início do período de expulsão, os profissionais de saúde deverão também rever os passos necessários ao bebê imediatamente após o parto. Essas ações essenciais após o parto, irão ajudá-lo numa transição bem-sucedida para a vida extrauterina. $O$ período de um minuto que se segue ao nascimento é chamado "minuto de ouro" para o bebê, pois este, para sobreviver, deverá começar a chorar ou a respirar aproximadamente no espaço de um minuto. Se o bebê não gritar nem respirar espontaneamente nesse espaço de tempo, os profissionais de saúde deverão então prestar-lhe rapidamente a devida assistência ${ }^{(6,7)}$.

O subitem que avalia se o bebê necessita ser aspirado, caso ele não esteja respirando, não se aplicou em nenhum parto observado, pois em todos eles o bebê respirou bem. No entanto, a prática de aspiração das vias aéreas é realizada rotineiramente em todos os recémnascidos na instituição hospitalar, mesmo nos casos em que estes não necessitem dessa prática.

O estudo denominado Nascer no Brasil, evidenciou que práticas consideradas inadequadas como uso de oxigênio inalatório $(9,5 \%)$, aspiração de vias aéreas $(71,1 \%)$ e gástrica $(39,7 \%)$ e uso de incubadora $(8,8 \%)$ foram excessivamente usadas ${ }^{(14)}$. Sendo assim, a aspiração orofaringeana e nasofaringeana deve ser realizada somente quando necessário e não deve ser uma prática sistemática do recém-nascido saudável (13).

Observou-se também que a disponibilidade de outro profissional para auxiliar teve $100 \%$ de adesão em ambos nos dois tipos de parto. Nesse contexto, a disponibilidade de um assistente durante o evento, se ocorrer uma situação de crise, é importante. Este poderá executar várias tarefas complementares, incluindo avaliar a mãe ou o bebê, iniciar fluidos endovenosos, administrar medicamentos, organizar transferências e pedir ajuda ${ }^{(7)}$.

Exposto às práticas que atingiram a totalidade da adesão, ressalta-se ainda os procedimentos que apresentaram déficit no manejo adequado ou não se aplicaram às situações, entres essas incluíram a antibioticoprofilaxia destinada à mãe no parto vaginal ${ }^{(7)}$. Apesar da taxa de adesão de $80 \%$ para essa ação no parto vaginal, as cinco mulheres que receberam antibióticos, estavam dentro dos critérios para inicio do tratamento.

Outros itens que obtiveram baixa adesão no parto vaginal foram as práticas de verificação de temperatura (56\%) e pressão (68\%). Notou-se que os técnicos de enfermagem da sala de parto seguem uma rotina de horários de seis em seis horas para a verificação dos sinais vitais, exceto quando a mulher apresenta alguma inter- 
corrência e necessita de monitorização dos sinais vitais em menor tempo. Ainda, a baixa adesão a esse procedimento pode ser explicada também pelo fato da equipe acreditar que o parto por ser normal e a mulher não ser submetida a cirurgia, os sinais vitais podem ser ignorados.

Nos itens que trazem informações sobre o uso correto do material essencial durante o parto, evidenciou que a prática de higienização das mãos após o parto obteve uma taxa de adesão de $60 \%$ no parto cesáreo e $76 \%$ no parto vaginal. Esse fato pode ser explicado devido os profissionais enfermeiros, que realizaram a maior parte dos partos normais, terem mais oportunidades de higiene das mãos e aderirem melhor a prática, em comparação com os profissionais médicos, responsáveis pela realização dos partos cesáreos ${ }^{(15)}$. Desse modo, a adesão a essa prática predominou no parto normal. Contanto, ressalta-se que o ideal seria que a higienização das mãos após o parto fosse realizada por todos os profissionais, alcançando uma taxa de adesão satisfatória de 100\%.

Um estudo transversal, descritivo com abordagem quantitativa realizado no município de Quixadá-CE com 48 profissionais de enfermagem, sobre higienização das mãos na assistência à saúde pela equipe de enfermagem, mostrou que a grande maioria desses profissionais realiza a higienização das mãos (83\%) conforme protocolo do Ministério da Saúde (16).

Diante disso, sabe-se que a higienização das mãos dos profissionais que lidam diretamente com o paciente, principalmente os profissionais da enfermagem, diminui sensivelmente a incidência e a gravidade das infecções hospitalares. Assim, a higiene das mãos é sem dúvida, a rotina mais simples, mais eficaz, de menor custo, além de maior importância na prevenção e controle da disseminação dessas infecções, devendo ser praticada por toda equipe, sempre ao iniciar e ao término de uma tarefa (17).

Em relação ao item que está associado ao uso de luvas durante o parto vaginal, foi alcançado $96 \%$ de taxa de adesão. $\bigcirc$ motivo para que tal prática não atingisse $100 \%$ de adesão, justificou-se devido a uma situação específica em que a mulher chegou na instituição hospitalar no período expulsivo e o profissional não teve tempo hábil para calçar as luvas.

O item que abrange a adesão em relação a administração de ocitocina à mãe um minuto após o parto, evidenciou uma taxa de adesão de $80 \%$ no parto vaginal. Os $20 \%$ de não adesão dessa prática no parto normal, justificou-se devido a necessidade da realização do procedimento de curagem nesses casos específicos.

Em relação aos resultados das taxas de adesão das práticas realizadas após o parto cesáreo e vaginal (no espaço de uma hora) trazidas no instrumento de observação da lista de verificação para partos seguros, destaca-se que a prática que está relacionada à verificação de hemorragia da mulher, obteve uma taxa de adesão de $100 \%$ no parto cesáreo e de $92 \%$ no parto vaginal.

De acordo com a Organização Mundial da Saúde (2017) ${ }^{(7)}$ a hemorragia pós-parto anormal é uma complicação grave que deverá ser detectada e tratada o mais cedo possível, por esse motivo, os profissionais devem estar atentos e observarem a mulher após o parto. Essa intercorrência pode ocorrer devido a várias razões, incluindo atonia uterina, retenção da placenta ou fragmentos da placenta, laceração vaginal ou rotura uterina.

Em relação à verificação dos sinais vitais (temperatura e pressão) das puérperas após o parto, constatou-se que no parto cesáreo a taxa de adesão foi 100\% para ambos os sinais vitais. Em contrapartida, no parto vaginal a taxa de adesão para verificar a pressão arterial foi de $52 \%$ e $40 \%$ para verificação de temperatura.

O período de pós-parto imediato é um período delicado em que a enfermagem deve ter um cuidado mais específico e criterioso. Deve-se dar ênfase nas duas primeiras horas, em que devem ser verificados a cada 15 minutos os sinais vitais, pois é nessa fase que ocorrem maiores casos de sangramentos, comprometendo assim o estado geral da paciente, podendo levar até ao óbito ou devem ser verificados os sinais vitais a cada 30 minutos com redução do tempo quando tiver necessidade ${ }^{(18)}$.

O item que verifica a necessidade da administração de antibiótico no bebê, obteve $100 \%$ de não adesão em ambos os partos (vaginal e cesáreo), pois o ideal é que o bebê não necessite dessa prática após o nascimento. O profissional que estiver preenchendo a Lista de Verificação deverá confirmar se o recém-nascido precisa de antibióticos logo após o parto (no espaço de uma hora) e, se for caso disso, os antibióticos deverão ser administrados de imediato. (7)

Os itens que avaliam a verificação dos sinais vitais (temperatura e frequência respiratória) do bebê após o nascimento, constataram que a taxa de adesão para essa prática foi de 15 (100\%) no parto cesáreo. No entanto, no parto vaginal, essa taxa foi de $92 \%$ para temperatura e $96 \%$ para frequência respiratória. Nos cuidados de enfermagem destacam-se a manutenção da temperatura corporal, em níveis normais, os quais podem ser alcançados por meio de atividades como: secar o $\mathrm{RN}$ ao nascer para evitar perdas de calor por evaporação, preparar o berço aquecido ou a incubadora dependendo da necessidade do neonato, aquecendo-o o mais rápido possível e avaliar as suas condições clínicas. Alguns procedimentos são responsáveis por promover a perda de calor como: exame clínico, banho e transporte. Esses procedimentos devem ser realizados juntamente com a monitorização dos sinais vitais, evitando assim complicações (19).

A prática que avalia a necessidade do bebê de cuidados especiais e monitorização obteve uma taxa de não 
adesão de $80 \%$ na cesariana e $68 \%$ no parto vaginal, pois o esperado é que o $\mathrm{RN}$ não necessite de tal prática. $\mathrm{O}$ instrumento deverá confirmar se o bebê precisa de cuidados especiais ou de monitorização logo após o parto (no espaço de uma hora) e, se necessário, deverá proceder de imediato a cuidados especiais ou de monitorização. Diante disso, os cuidados especiais ou monitorização deverão ser proporcionados ao bebê se este tiver nascido mais de um mês antes do final da gestação, se tiver um peso ao nascer de $<2500$ gramas, se precisar de antibióticos, ou exigir reanimação para o ajudar a chorar ou respirar ao nascer ${ }^{(7)}$.

A prática relacionada à amamentação no espaço de uma hora após o nascimento, resultou numa taxa de adesão de $64 \%$ no parto normal e no parto cesáreo não foi observada essa prática, o que evidencia que o tipo de parto cesáreo não contribuiu para que essa prática seja realizada. Em contrapartida, um estudo descritivo desenvolvido em uma instituição hospitalar com Iniciativa Hospital Amigo da Criança da Tríplice Fronteira, realizado com 88 binômios, que teve como objetivo identificar a prevalência da amamentação na primeira hora de vida e seus resultados para a manutenção do aleitamento materno, evidenciou que a amamentação na primeira hora de vida iniciou em $82,8 \%$ dos nascimentos por parto normal e em $78 \%$ dos nascimentos por parto cesáreo. Nesse estudo, o parto vaginal foi tido como um fator positivo para a amamentação na primeira hora, apesar de a diferença estatística apresentar-se pequena, cerca de $4 \%{ }^{(20)}$.

A amamentação no espaço de uma hora após o nascimento, ajuda o bebê a estabelecer uma boa ligação com a mãe e pode ainda estimular a contração uterina da mãe através dos hormônios maternos liberados, evitando a hemorragia após o parto ${ }^{(7)}$.

Quanto ao item que avalia se o bebê foi colocado em contato pele a pele com a mãe logo após o nascimento, obteve uma taxa de adesão de $92 \%$ no parto vaginal e $26,6 \%$ no parto cesáreo, constatando que essa prática teve melhor adesão por parte dos profissionais que realizaram os partos normais. Nesse sentido, pode-se dizer que o tipo de parto normal pode estimular o contato pele a pele com mãe logo após o nascimento.

Estudo observacional prospectivo que utilizou a lista de verificação para parto seguro da OMS, obteve $68,7 \%$ de adesão para as práticas de amamentação e contato pele a pele logo após o parto (21).

Nesse âmbito, os bebês são particularmente sensíveis ao estresse do frio e podem surgir rapidamente complicações se a sua temperatura corporal descer abaixo do limite normal. Portanto, o contato com a pele da mãe é o melhor método para mantê-lo aquecido, além de ajudar a promover a ligação entre o bebê e a mãe ${ }^{(7)}$. As Diretrizes Nacionais de Assistência ao Parto Normal (2017), assim como a OMS, recomendam estimular as mulheres a terem contato pele-a-pele imediato com a criança logo após o nascimento ${ }^{(13)}$.

O item que aborda a prática de explicar os sinais de perigo para as mães ou acompanhantes, evidenciou que no parto normal a taxa de adesão a essa prática foi de $28 \%$ e no parto cesáreo foi de $6,66 \%$. Em contrapartida a esses achados, o estudo observacional prospectivo, realizado no Sri Lanka, evidenciou que 449 (54,5\%) profissionais confirmaram que a mãe e/ou acompanhante irá pedir ajuda se houver sinais de perigo ${ }^{(21)}$.

Segundo a Organização Mundial de Saúde (2017) as complicações são imprevisíveis e podem surgir a qualquer momento durante o processo do nascimento, tanto para as mães como para os bebês. As mães (e acompanhantes) podem ser ensinados a reconhecer os sinais de perigo e a alertar os profissionais de saúde de imediato, caso esses sinais ocorram. Os sinais de perigo que a mulher pode desenvolver no período pós-parto são, hemorragia, forte dor abdominal, forte dor de cabeça ou distúrbio visual, respiração difícil, febre e calafrios ou dificuldade em urinar ${ }^{(7)}$.

Diante dos resultados desse estudo, ressalta-se a importância de ampliar as pesquisas sobre a lista de verificação de parto seguro nas maternidades brasileiras, tendo em vista que esse tipo de investigação, além de mostrar um diagnóstico das ações, despertam os profissionais e gestores quanto a importância de adotar as práticas seguras durante $o$ parto.

\section{CONCLUSÃO}

Diante dos resultados, pode-se concluir que as taxas de adesão às práticas avaliadas pelo instrumento foram satisfatórias na maioria dos itens. Os profissionais tendem a aderir mais as práticas seguras no parto cesáreo, em detrimento ao parto vaginal, talvez pelo primeiro caracterizar-se por um procedimento cirúrgico. No entanto, observou-se que a interação do bebe com a mãe é mais estimulada no parto vaginal.

Além disso, o instrumento destacou-se como uma estratégia que pode fortalecer a cultura de segurança do paciente na instituição, pois permite trabalhar mais a adesão dos profissionais às práticas seguras.Vale salientar que o instrumento não tem a finalidade de julgar as práticas dos profissionais avaliados, e sim, de coletar informações em relação à assistência prestada, buscando melhorar cada vez mais a qualidade dos cuidados prestados.

Portanto, recomenda-se a realização de estudos futuros com a aplicação do instrumento de observação da lista de verificação para partos seguros nas instituições hospitalares, e através dos resultados da aplicação, o desenvolvimento de intervenções, abordando a importância das práticas seguras, principalmente aquelas que não obtiveram boa adesão por parte dos profissionais. 


\section{REFERÊNCIAS}

I. Reis LGC, Pepe VLE, Caetano R. Maternidade segura no Brasil: o longo percurso para a efetivação de um direito. Physis (Rio J). 20 I I;2 I (3): I I39-59.

2. Diniz CSG. Implementação da presença de acompanhantes durante a internação para o parto: dados da pesquisa nascer no Brasil. Cad Saúde Pública. 20I4; 30(I): 40-53.

3. Gomes ARM, Pontes DS, Pereira CCA, Brasil AOM, Moraes LCA. Assistência de enfermagem obstétrica na humanização do parto normal. Revista Recien - Revista Científica de Enfermagem. 20 I4;4(I I):23-27.

4. World Health Organization. Trends in maternal mortality: 1990 to 2015 Estimates by WHO, UNICEF, UNFPA, World Bank Group and the United Nations Population Division. $2010.44 p$

5.Organização Mundial da Saúde. Lista de Verificação da OMS para Partos Seguros. [Internet]. OMS; 2018 [acesso 2019 abr 23]. Disponível em: https://www.who.int/patientsafety/ implementation/checklists/childbirth_portuguese/en/

6. Organização Mundial da saúde. Guia de Implementação da Lista de Verificação da OMS para Partos Seguros: melhorar a qualidade dos partos realizados em unidades de saúde para as mães e os recém-nascidos. Genebra, 2017.

7.World Health Organization. Safe Childbirth Checklist Programme: an overview. Geneva:World Health Organization; 2013.

8. Domingues RMSM, Dias MAB, Pereira MN, Torres JÁ, d'Orsi E, Pereira APE et al. Processo de decisão pelo tipo de parto no Brasil: da preferência inicial das mulheres à via de parto final. Cad. Saúde Pública 20 I4; 30 Supl: S I0I-I I6.

9. Bagheri A, Masoodi-Alavi N, Abbaszade F. Effective factors for choosing the delivery method among the pregnant women in Kashan. Feyz 2012, 16(2): 146-I53. Acesso em: 2019 abr 23. Disponível em http://feyz.kaums.ac.ir/browse. php?a_id $=\mid 428 \&$ sid $=\mid$ \&s $\mid$ c_lang=en

10. Patabendige M, Senanayake H. Implementação do programa de lista de verificação de parto seguro da OMS em um ambiente de atenção terciária no Sri Lanka: uma experiência de país em desenvolvimento. Gravidez e Parto do BMC. 20I5; I5 (12): 33-40.

I I. da Silva, T. M., Oliveira, N. de S., do Prado, P. R. Cirurgias seguras: instrumento de enfermagem obstétrica perioperatória. South American Journal of Basic Education, Technical and Technological ,6(I). Disponível: Recuperado de https:// periodicos.ufac.br/index.php/SAJEBTT/article/view/2219
12. Oliveira AC, Gama CS. Antissepsia cirúrgica e utilização de luvas cirúrgicas como potenciais fatores de risco para contaminação transoperatória. Esc Anna Nery, 20 I 6; 20(2): 370-377.

13. Diretriz Nacional de Assistência ao Parto Normal. Brasília, DF: MS, 2016. Disponível em: <http://conitec.gov.br/ images/Consultas/20 I6/Relatorio_Diretriz-PartoNormal _CP.pdf>.Acesso em : 10 out 2017.

14. Moreira MEL, Gama SGN, Pereira APE, Silva AAM, Lanksy S, Pinheiro RS et al. Práticas de atenção hospitalar ao recémnascido saudável no Brasil. Cad. Saúde Pública,20I4; 30(I): 128-139.

15. Primo MGB, Ribeiro LCM, Figueiredo LFS, Sirico SCA, Souza MA. Adesão à prática de higienização das mãos por profissionais de saúde de um Hospital Universitário. Rev. Eletr. Enf. [Internet]. 2010 abr./jun.; I2(2):266-7I. Disponível em: http://dx.doi.org//0.52 I6/10.52 /6/ree.v I2i2.7656.

16. Gomes RKG, Cândido RL, Maniva SJC de F, Holanda RE. Segurança do paciente: higienização das mãos na assistência à saúde pela equipe de enfermagem. Revista Expressão Católica Saúde. 2017; 2(2): 69-75.

17. Sales DCR, Wingester ELC, Rodrigues TRS. Concepções dos profissionais de enfermagem de uma unidade de centro cirúrgico sobre infecção hospitalar e lavagem de mãos antes e após a aplicação de uma atividade educativa. Revista Digital FAPAM. 2016; 2(2): 99-109.

18. Gomes GF, Santos APV. Assistência de enfermagem no puerpério. REv Enfe Contemporânea.v. 6, n. 2 (2017) http:// dx.doi.org// 0.17267/23 I7-3378rec.v6i2.1407

19. Rolim KMC, Freitas LC, Lima A de H G, Magalhães FJG, Pessoa E. Rev. Enf. Ref. [online]. 2015;(6): 9-I6. http://dx. doi.org// 0.I2707/RIVI4070.

20. Netto A, Spohr FA, Zilly A, França AFO, Brischiliari SCR, Silva RMM.Amamentação na primeira hora de vida em uma instituição com iniciativa hospital amigo da criança. Cienc Cuid Saude, v. I5, n. 3, p. 515-521, jul./set., 2016.

21. Patabendige M, Senanayake H. Implementação do programa de lista de verificação de parto seguro da OMS em um ambiente de atenção terciária no Sri Lanka: uma experiência de país em desenvolvimento. Gravidez e Parto do BMC. 20I5; I5 (I2): 33-40.

Recebido: 2019-1 |-26

Aceito: 2020-03-02 